\title{
Interstitial Boron Atoms in the Palladium Lattice of an Industrial type of Nano-catalyst: Properties and Structural Modifications
}

\author{
Tianyi Chen $¥ \S$, Ieuan Ellis ${ }^{\ddagger} \%$, Thomas J.N. Hooper ${ }^{\nabla}$, Emanuela Liberti§, Lin Ye ${ }^{\ddagger}$, Benedict T. W. Lo \\ Colum O’Leary§, Alexandra A. Sheader\$, Gerardo T. Martinez§, Lewys Jones§, Ping-Luen $\mathrm{Ho}^{\ddagger \S}, \mathrm{Pu}$ \\ Zhao ${ }^{\ddagger}$, James Cookson\%, Peter T. Bishop\%, Philip Chater ${ }^{\perp}$, John V. Hanna ${ }^{\nabla *}$, Peter Nellist ${ }^{\S *}$ and Shik \\ Chi Edman Tsang ${ }^{\sharp *}$
}

\author{
‡ Wolfson Catalysis Centre, Department of Chemistry, University of Oxford, Oxford, OX1 3QR, UK \\ ${ }^{\nabla}$ Department of Physics, University of Warwick, Coventry, CV4 7AL, UK \\ $\S$ Department of Materials, University of Oxford, Oxford, OX1 PH, UK \\ ${ }^{\perp}$ Diamond Light Source Ltd, Harwell Science and Innovation Campus, Didcot, Oxfordshire, OX11 0DE, UK \\ \% Johnson Matthey, Blount's Court, Sonning Common, Reading, RG4 9NH, UK
}

KEYWORDS Nanomaterial, electron microscopy, crystal structure

\begin{abstract}
It is well established that the inclusion of small atomic species such as boron (B) in powder metal catalysts can subtly modify catalytic properties, and the associated changes in the metal lattice implies that the B atoms are located in the interstitial sites. However, there is no compelling evidence for the occurrence of interstitial B atoms, and there is a concomitant lack of detailed structural information describing the nature of this occupancy and its effects on the metal host. In this work, we use an innovative combination of high-resolution ${ }^{11} \mathrm{~B}$ magic-angle-spinning (MAS) and ${ }^{105} \mathrm{Pd}$ static solid state NMR nuclear magnetic resonance (NMR), synchrotron X-ray diffraction (SXRD), in-situ X-ray pair distribution function (XPDF), scanning transmission electron microscopy-annular dark field imaging (STEM-ADF), electron ptychography and electron energy loss spectroscopy (EELS) to investigate the B atom positions, properties and structural modifications to the palladium lattice of an industrial type interstitial boron doped palladium nanoparticle catalyst system (Pd-intB/C NPs). In this study we report that upon B incorporation into the Pd lattice, the overall face centered cubic (FCC) lattice is maintained, however short range disorder is introduced. The ${ }^{105} \mathrm{Pd}$ static solid-state NMR illustrates how different types (and levels) of structural strain and disorder are introduced in the nanoparticle history. These structural distortions can lead to the appearance of small amounts of local hexagonal close packed (HCP) structured material in localized regions. The short range lattice tailoring of the Pd framework to accommodate interstitial B dopants in the octahedral sites of the distorted FCC structure can be imaged by electron ptychography. This study describes new toolsets that enables the characterization of industrial metal nanocatalysts across length scales from macro-analysis to micro-analysis, which gives important guidance to structure-activity relationship of the system.
\end{abstract}

\section{INTRODUCTION}

The introduction of dopants into metal framework catalysts is known to modulate the electronic and structural properties, giving rise to accelerated conversion rates, increased selectivities ${ }^{1}$ and enhanced stabilities of some specific catalytic reactions ${ }^{2}$. With regard to the site modification of the host unit cell, doping strategies can be divided into substitution and interstitial mechanisms. Substitution doping describes the replacement of an atom in the host unit cell by a foreign species; for example, in a system such as $\mathrm{BiO}_{1-\mathrm{x}} \mathrm{S}_{\mathrm{x}-}$ $\mathrm{CuSe}$, some oxygen atoms in the host BiOCuSe framework are replaced by sulphur ${ }^{3}$. Interstitial doping depicts small foreign atoms of light elements taking residence at the interstitial sites within the host metal unit cell.4,5 Iron carbide is a typical example of this phenomenon where the carbon (C) atoms reside within the interstitial positions of the metallic Fe framework. ${ }^{6}$ Once inserted, these small atoms can modify the geometry and electronic structure of the host metals, which has a direct impact on its Fermi level and density of states, thus allowing the metal to be potentially tuned for catalytic applications ${ }^{7}$. For catalysis scenarios, the main advantage of the latter interstitial doping scheme is that the lighter atoms incorporated into the metal atom framework can exert electronic and structural modifications to the host metal without direct involvement or interference to the surface catalytic properties. It has been shown that the addition of small atoms such as B or C to Fe or Co during synthesis can enhance the catalytic activities and extend stability in Fischer-Tropsch reactions ${ }^{2,8}$. Very recently, doping with B atoms was shown to dramatically increase the average oxidation state of copper metal hence achieving the highest reported selectivity of electrocatalytic reduction of $\mathrm{CO}_{2}$ to $\mathrm{C}_{2} \mathrm{H}_{4}$ at $70 \%{ }^{9}$. However, many of the synthesis routes for in- 
terstitial doping of industrial- type of metallic based nanoparticles (NPs) do not lead to homogeneously distributed dopants in the metal lattice, rendering the characterization of this phase difficult.

In order to correlate the structure-activity relationships of the doped metal catalysts, an important task in characterization is to give local and global assessments of structural changes using appropriate techniques. Traditionally, single crystal diffraction has been utilized for these investigations which provides average atomic positions within the catalyst structure. However, single crystal models may not be a good representation for industrial catalysts since these contain not only structural and grain boundary defects, but also non-uniform distribution of dopants across volume of the NPs. It thus remains challenging to represent real industrial catalysts with accurate models of their average and localized solid state structures despite the developments of some useful crystal growth techniques ${ }^{10}$.

The conventional structural characterization of industrial powder catalyst samples upon interstitial doping of metal NPs relies on laboratory based X-ray powder diffraction (XRD) ${ }^{5}$, X-ray photoelectron spectroscopy (XPS) ${ }^{11}$ and high resolution transmission electron microscopy (HRTEM) ${ }^{12}$ which can provide generic structural information, surface composition and local structure. Although image contrast quantification of the HRTEM images may in principle be applied to identify all types of atoms, in practice the low compositional dependence and additional contrast arising from the non-uniform support of catalyst limits interpretability ${ }^{13}$. Recently, density function theory calculations (DFT) ${ }^{14}$ have provided a very useful toolset to model the atomic positions and electronic modifications on fundamental catalytic processes. However, these conventional analytical techniques and DFT calculations provide limited answers to key questions relevant to interstitial modification of light elements in supported metal NPs. For example, how is the local modification influencing the nearby parent structure? Can we visualize the interstitial dopant on the multicomponent polycrystalline industrial powder catalyst at the atomic level?

This study demonstrates that, in addition to conventional techniques, an innovative combination of high resolution ${ }^{11} \mathrm{~B}$ solid state magic-angle-spinning nuclear magnetic resonance spectroscopy (MAS NMR) ${ }^{15,16,17}$ and broadline ${ }^{105} \mathrm{Pd}$ solid state static NMR ${ }^{18}$, synchrotron X-ray powder diffraction (SXRD) ${ }^{19}$, aberration-corrected scanning transmission electron microscopy-annular dark-field (STEM-ADF) imaging $^{20}$, in-situ X-ray pair distribution function (XPDF) ${ }^{21}$, electron energy loss spectroscopy (EELS) ${ }^{22}$ and electron ptychography ${ }^{23}$ to characterize Pd-intB/C NPs system. This industrial-type powder catalyst was chosen as a case study for the investigation of Pd host unit cell structural modification and B atom location. This catalyst sample has been demonstrated to show premium activities in formic acid decomposition $^{24}$, and selective hydrogenation of acetylene and phenylalkyne 25,26 . The role of the interstitial B is expected to downshift the $\mathrm{d}$ band centre of the Pd host system due to the overlap of B (s, p) and Pd (d) orbital, allowing the tuning of the chemical adsorption between metallic surface and chemical species.
The structural model provided from the Rietveld refinement of high quality SXRD was compared with the combined results of XPDF and STEM-ADF imaging, eventually converging upon a reliable unit cell model. Recently, XPDF has proved powerful in structural determination of both crystalline and amorphous materials at high precision ${ }^{27-30}$, and it represents a valuable tool in this overall approach. The developments in STEM-ADF imaging digital processing allow scanning distortions to be alleviated or even fully corrected ${ }^{31}$. In addition, EELS allows analysis of local elemental distributions in small selected areas, which can be combined with electron ptychography to image the dopants. The principle of electron ptychography is to use a highly convergent electron beam to form a focused probe and then to record the resultant diffraction patterns formed by transmitted electrons in the far field ${ }^{32}$. The phase information can be retrieved with regards to the interference of direct and diffracted beams. More recently, developments in fast-pixelated detectors and data processing have allowed to yield simultaneous $\mathrm{ADF}$ and phase imaging for detection of both light and heavy elemental species. So far, this technique has been successfully applied to carbon nanotubes (CNTs) ${ }^{23}$ and light elements in crystalline materials ${ }^{33}$.

In this study, we report evidence that doping B species into industrially-relevant powdered Pd metal NP systems generates sub-surface B positions within the Pd metal framework of the NP. This work presents the first ptychography measurements having visualized the interstitial $\mathrm{B}$ atoms within the distorted octahedral holes of the Pd FCC structural framework; these observations have been correlated with short range information on speciation and disorder provided by ${ }^{11} \mathrm{~B}$ solid state MAS NMR and ${ }^{105} \mathrm{Pd}$ solid state NMR; this technique is particularly suited to the observation of the metal positions in catalytic systems ${ }^{34}$. In addition, these metastable sub-surface B species could create severe distortion to the metal host subsequently producing minor HCP phases. At elevated temperatures, these systems exhibit thermal instability and decompose back to the host metal lattice and surface B species. It is believed that this study describes a new characterization toolset necessary for the long range and short-range structural evaluation of interstitial dopants in supported industrial metal nanocatalysts.

\section{RESULTS AND DISCUSSION}

Initial characterization of $\mathbf{P d}{ }^{-{ }^{i n t}} \mathbf{B} / \mathbf{C}$ NPs. $\mathrm{Pd}$-intB/C was made according to a previously reported synthetic strategy involving the thermal decomposition of borane hydride THF complex in the presence of $\mathrm{Pd} / \mathrm{C}$ at $200^{\circ} \mathrm{C}^{26}$. This method produced relatively monodisperse Pd NPs with an average size of $6.69 \mathrm{~nm}$, as determined by ADF-STEM shown in Supplementary Information Figure S1. The structural modification to $\mathrm{Pd}$ lattice can be seen from powder X-ray diffraction (PXRD) patterns shown in Figure 1a. As expected, the Pd/C presents reflections that match the FCC structure of $\mathrm{Pd}$ metal. Five diffraction peaks of Pd NPs are observed at $2 \theta=$ $39.97^{\circ}, 46.51^{\circ}, 68.08^{\circ}, 81.92^{\circ}$ and $86.42^{\circ}$ respectively, corresponding to the (111), (200), (220), (311) and (222) indexes of the FCC structure. The subsequent PXRD characterization of $\mathrm{Pd}-{ }^{-i n t} \mathrm{~B} / \mathrm{C}$ showed that while similar data was acquired, a close inspection reveals small but well defined 
shifts of $\sim 1-3^{\circ}$ lower $2 \theta$ in the diffraction peak positions. The (111) diffraction peak shifts from $2 \theta=39.97^{\circ}$ to $38.71^{\circ}$; the (200) diffraction peak shifts from $2 \theta=46.51^{\circ}$ to $44.99^{\circ}$; the (220) diffractions peak shifts from $2 \theta=68.08^{\circ}$ to $65.65^{\circ}$; the (311) diffraction peak shifts from $2 \theta=81.92^{\circ}$ to $79.06^{\circ}$ and the (222) diffraction peak shifts from $2 \theta=86.42^{\circ}$ to $83.62^{\circ}$, giving rise to average expanded $d$-spacing $\left(d_{111}=\right.$ $2.33 \AA ; \mathrm{d}_{200}=2.01 \AA ; \mathrm{d}_{220}=1.42 \AA ; \mathrm{d}_{311}=1.21 \AA ; \mathrm{d}_{222}=1.16$ $\AA$ ), compared with $\mathrm{d}_{111}=2.25 \AA$; $\mathrm{d}_{200}=1.95 \AA$; $\mathrm{d}_{220}=1.38 \AA$; $\mathrm{d}_{311}=1.17 \AA ; \mathrm{d}_{222}=1.12 \AA$ respectively for pure Pd metal NPs. The afforded lattice parameter is now $4.04 \AA$, thus representing increase of $\sim 3.9 \%$. This systematic shift to lower $2 \theta$ values are indicative of the expected lattice expansion of the $\mathrm{Pd}$-int $\mathrm{B} / \mathrm{C}$ system when $\mathrm{B}$ is incorporated. Note that larger degrees of lattice expansion are anticipated closer to the surface of the supported Pd NPs due to the low temperature synthesis used.
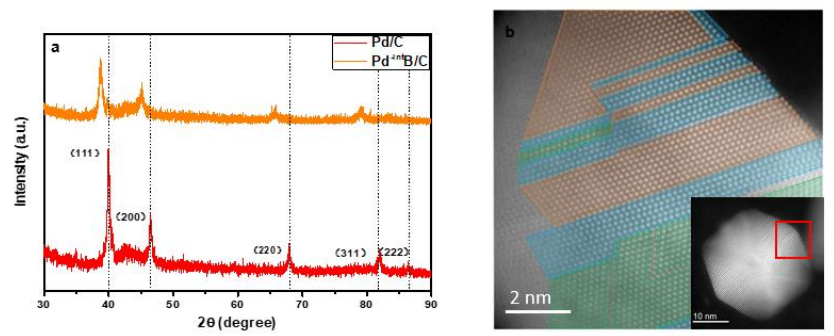

Figure 1. Experimental evidence for the interstitial effect of $\mathrm{Pd}$-intB/C (a) Wide-angle PXRD patterns of Pd/C (red), Pdint $\mathrm{B} / \mathrm{C}$ (yellow). The peaks labelled with dash lines corresponding to Pd (111), (200), (220), (311) and (222), characteristic of the $\mathrm{Pd}$ face-centred cubic (FCC) structure. ${ }^{35}$ Shifting to lower angles in the case of $\mathrm{Pd}$-intB/C (orange) indicates lattice expansion. (b) STEM-ADF image of a Pd-intB/C NPs, orange: FCC region of [110], green: FCC region of [110] twinned with respect to the orange region: blue: HCP region of [100].

Further verification of the successful incorporation of B atoms into interstitial sites of Pd is observed through the absence of the typical $\beta$-hydride desorption peak from temperature programmed reduction (TPR) of the sample (Supplementary information Figure S2). This peak arises at 75 ${ }^{\circ} \mathrm{C}$ when $\mathrm{H}$ atoms diffuse out from octahedral sites from subsurface region of Pd NPs after $\mathrm{H}_{2}$ dissociation and $\mathrm{H}$ migration at room temperature on the surface of Pd NPs in an $\mathrm{H}_{2}$ atmosphere ${ }^{36}$. The lack of this $\beta$-hydride peak indicates that the interstitial lattice sites of Pd near the particle sub-surface are already occupied by the B atoms due to the stronger interaction, denying access to hydrogen atoms.

Although XRD shows that the bulk phase of supported PdintB NPs fits well with an expanded Pd FCC structure, the STEM-ADF image (Figure 1b) obtained using multi-frame acquisition and subsequent rigid and non-rigid registration $^{37}$ highlights a selected region of a Pd-intB NP where HCP and FCC structures are distributed irregularly at the particle edge region. This reveals that the presence of doping can result in a local phase transition of FCC to HCP to a small extent, presumably within the same particle due to the high local lattice strain imposed by the B doping. This phenome- non has also been observed by Kobayashi et. al, where excess dopant source was shown to result in the transformation of FCC to $\mathrm{HCP}^{38}$.

Pd-int $\mathbf{B} / \mathbf{C}$ phase identification. In order to identify potential phases in this sample and determine their lattice parameters with confidence, high-resolution SXRD data $(\lambda=$ $0.97920 \AA$ ) was employed (See supplementary information, Figure S3 and Figure S4, Table S1 and Table S2 for details). Le Bail fitting was performed in TOPAS Academic 5.039. The possible phases, (Pd/C, Pd-intB/C (FCC: Fm-3m) and PdintB/C (HCP: P6mm), were introduced sequentially. R-factors $\left(R_{w p} / R_{p} / R_{\text {exp }} / \chi 2\right)$ were used to gauge the quality of fitting. With the progressive increase in the number of structures, the R-factors were found decreasing, which indicated that the use of $\mathrm{Pd} / \mathrm{C}, \mathrm{Pd}$-intB/C $(F m-3 m)$ and $\mathrm{Pd}$ intB/C $(P 6 \mathrm{~mm})$ was approaching to the real structure (Supplementary Information Table. S1). This is in good agreement with the STEM-ADF image (Figure 1b). The space group of the FCC and HCP structure was Fm-3m and $P 6 \mathrm{~mm}$, respectively. From the Le Bail fitting described, lattice parameters of $a=\mathrm{b}=\mathrm{c}=3.99(1) \AA$ were obtained for the FCC phase, an increase of $2.6 \%$ in comparison to that of Pure $\mathrm{Pd} / \mathrm{C}(3.890 \AA)$. The HCP unit cell lattice parameter was determined to be $a=2.87(1) \AA$ and $c=3.62(5) \AA ̊$ respectively (Supplementary Information Table. S2).

In-depth investigations of local structure distortions. Although preliminary STEM-ADF images and SXRD data can identify the presence of the small extent of the HCP phase within a majority FCC phase, they are unable to give an estimate of this phase composition ratio. Furthermore, the other relevant refinement techniques are also not appropriate due to peak boarding and an insufficient number of the diffraction peaks measured from the powdered industrial sample.
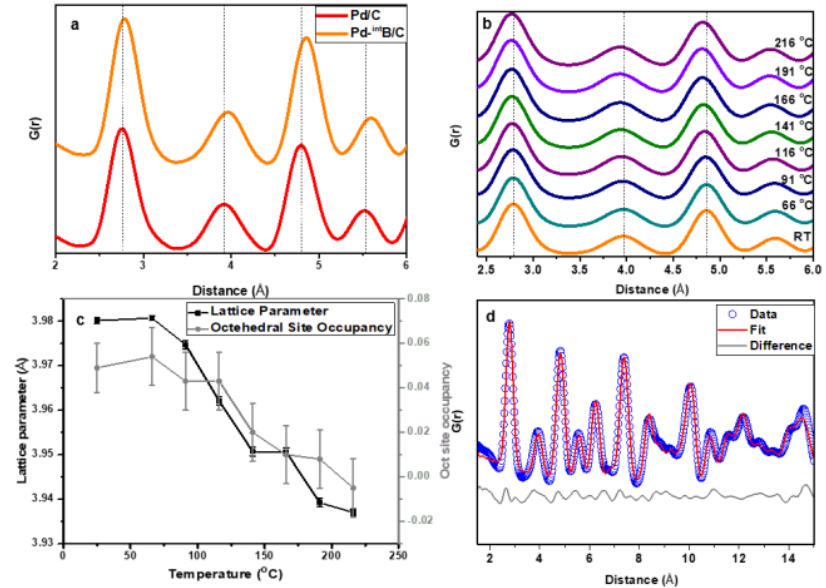

Figure 2. Probe local structural distortion by using in-situ XPDF and PDFgui. (a) XPDF analysis of Pd/C and Pd-intB/C at room temperature provides data on $\mathrm{Pd}-\mathrm{Pd}$ bond distance, after zoning up in the range of 2 to $6 \AA$. (b) in-situ XPDF of Pdint $B / C$ from room temperature to the elevated temperature, after zoning up at the range of 2 to $6 \AA$. (c) The Pd-Pd lattice parameter and corresponding $B$ occupancy in the octahedral sites against temperature. (d) A typical fit - fitting range $=1.5-15$ $\AA$. $\mathrm{R}_{\mathrm{w}}=12.5 \%$; other fittings parameters can be found in the SI.

The XPDF technique (utilizing a synchrotron source) was used to elucidate a more detailed characterization of the $\mathrm{Pd}$ 
structure with and without B present as an interstitial dopant. As seen in the G(r) of Pd/C (Figure 2a), a clear set of FCC peaks can be seen, which fits the characteristic FCC structure of Pd lattice. Notably, the second peak at around 3.9027(7) Å can be assigned to the Pd FCC lattice (parameter, a), which agrees well with the literature value ${ }^{40}$. The FCC structure pattern is also observed in the G(r) of PdintB/C, with a lattice expansion $(a=3.9801(7) \AA ̊)$ when compared to the pure $\mathrm{Pd} / \mathrm{C}$ (which is coincident with the structural information of interstitial FCC phase retrieved by the TOPAS refinement- see Supplementary information Table S2). However, as XPDF directly measures the bulk lattice structure of Pd, an indicative Pd HCP lattice peak cannot be observed, thus suggesting that NPs are clearly dominated by FCC phase, whereas HCP structure is only a minor component. The thermal stability of $\mathrm{Pd}$-int $\mathrm{B} / \mathrm{C}$ phases have also been studied with in-situ heating in the XPDF (Figure 2b). The lattice parameter, $a$, gradually decreases from 3.9801(7) A (at room temperature) to 3.9370(7) A (at 216 ${ }^{\circ} \mathrm{C}$ ) (see the temperature-programmed G(r) analyses from the in-situ XPDF measurement in Figure 2c). This suggests that the expanded Pd FCC lattice returns to its initial value presumably due to the thermally driven exclusion of the interstitial B atoms from the Pd lattice.

Furthermore, the PDFgui software was used to probe the presence of $B$ atoms in the bulk Pd lattice. As all of the temperature programmed G(r) XPDF data display a typical FCC pattern (from room temperature to $216^{\circ} \mathrm{C}$ ), there are only two possible sites where $B$ could reside, namely the octahedral sites (with the fractional atomic coordinates of $[0,0$, $1 / 2$ ) and the tetrahedral sites (with the fractional atomic coordinates of $(1 / 4,1 / 4,1 / 4))$. According to the radius ratio rules, the maximum limiting radius of the tetrahedral sites is $67 \mathrm{pm}$ which is too small for B (85 pm) to reside in (See Supplementary Information, Figure S5 and section 7 for calculation). We, therefore, fitted our XPDF data using a Pd FCC model with B in the octahedral sites (Figure 2d). The refined atomic parameters data were listed in Table S3 of the Supplementary Information. Note that the $\mathrm{R}_{\mathrm{w}}$ factors fall within the acceptable region 10-20\%. From room temperature to $66{ }^{\circ} \mathrm{C}$, the lattice parameter maintains at about 3.9801(7) $\AA$ and the site occupancy of B in the octahedral sites is about $5 \%$. When the sample is further heated, both the lattice parameter and B site occupancy begin to fall. The refinement results show that the $B$ site occupancy is almost zero at $191^{\circ} \mathrm{C}$. The leaching out of $\mathrm{B}$ at such low temperature indicates that $\mathrm{B}$ is possibly only kinetically stable in the interstitial octahedral sites at room temperature, but that most of B leaves from the interstitial sites of the NPs presumably with sufficient kinetic energy to relax the strained sites at elevated temperatures.

Solid State NMR analysis. The presence of B in the interstitial octahedral sites is also confirmed by the high-resolution solid-state NMR, which is a technique that is sensitive to differences in short-range chemical environments.
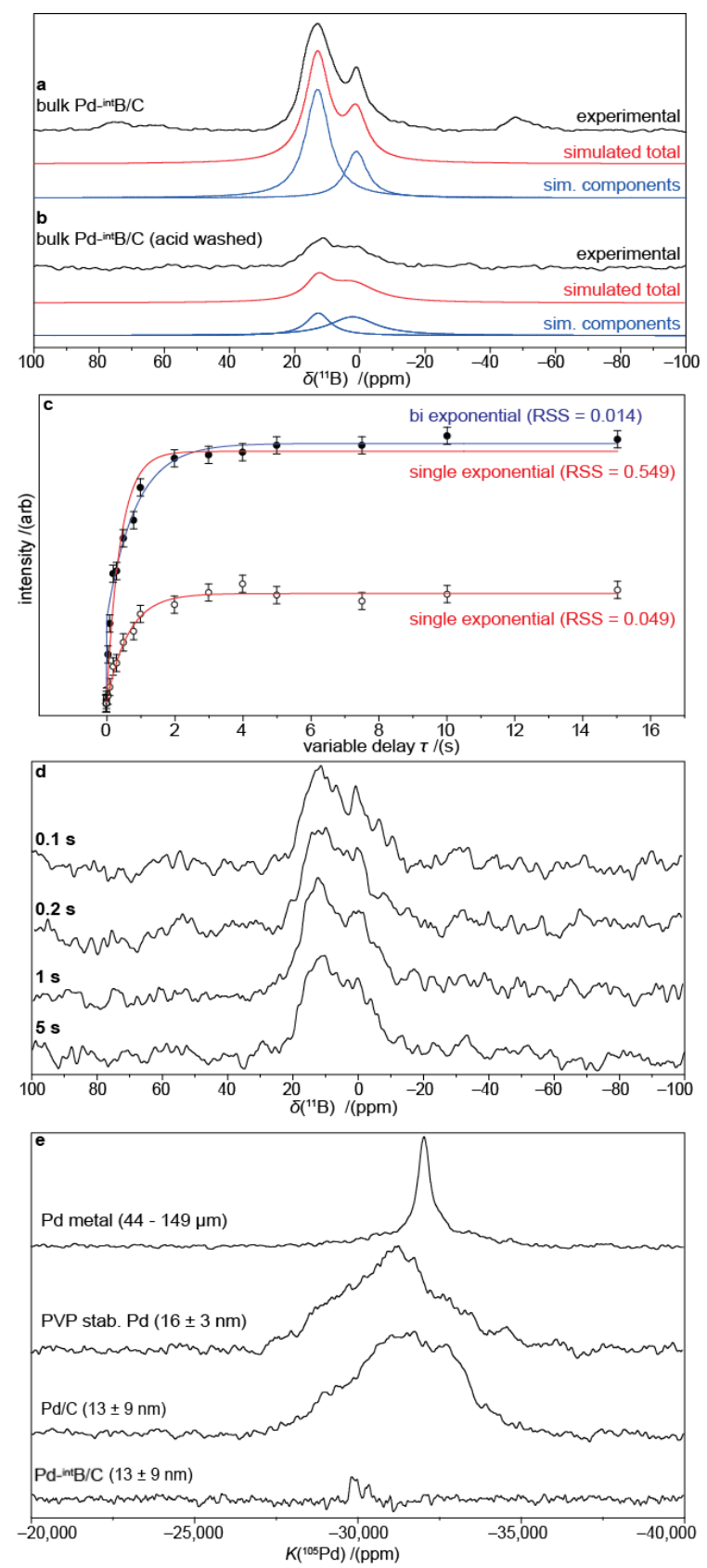

Figure 3. (a) ${ }^{11} \mathrm{~B}$ MAS NMR data of $\mathrm{Pd}$-intB/C before acid washing, and (b) after acid washing, where the experimental spectra are in black and the deconvolved simulations are in red (simulation total) and blue (individual components). (c) the fitted ${ }^{11 B}$ saturation recovery $\mathrm{T}_{1}$ relaxation measurements for the two resolved resonances at $12.6 \mathrm{ppm}$ (solid circles) and $0.7 \mathrm{ppm}$ (hollow circles), where single exponential and bi-exponential fits are represented by red and blue lines, respectively, and the residual sum of squares of each fit is given. (d) variable recycle delay ${ }^{11 B}$ MAS NMR experiment on the Pd-intB/C system after the acid washing treatment showing the predominance of the fast-relaxing component in these data. (e) ${ }^{105} \mathrm{Pd}$ VOCS static NMR comparing Pd-intB/C to Pd metal, PVP stabilised nanoparticles and the $\mathrm{Pd} / \mathrm{C}$ precursor labelled with their respective particle size distributions.

High resolution ${ }^{11} \mathrm{~B}$ MAS NMR can resolve and differentiate closely related B species within the Pd NP matrix with characteristic chemical shifts $(\delta)$ and spin-lattice $\left(T_{1}\right)$ relaxation 
parameters. The ${ }^{11} \mathrm{~B}$ MAS NMR data in Figure 3 of Pd-intB/C before acid washing (Figure $3 a$ ), and after acid washing (Figure 3b), where the experimental spectra are in black and the deconvolved simulations are in red. They show resonances centered at $12.6 \mathrm{ppm}$ and $0.7 \mathrm{ppm}$. Correlated with previous characteristic measurements, it is presumed that one of the resonances represents the interstitial B species and the other can be attributed to surface B contaminants.

To identify which resonance corresponds to the interstitial $\mathrm{B}, T_{1}$ relaxation measurements were performed using the saturation recovery method ${ }^{41}$. Single-exponential, bi-exponential and stretched-exponential (not shown but listed in Table S4) fits were attempted on the $T_{1}$ data shown in Figure 3c, and the residual sum of squares (RSS) for each fit has been provided as a measure of the discrepancy between the model and the data ${ }^{42}$. The ${ }^{11} \mathrm{~B}$ MAS NMR spectrum in Figure 3a was initially deconvolved into the two resonances and the relaxation measurements are listed in Supplementary Information Table S4. The relaxation data of the resonance centered at $0.7 \mathrm{ppm}$ is adequately simulated by a single exponential yielding a $T_{1}$ of $0.64 \pm 0.04 \mathrm{~s}$ (low RSS of 0.049 ). In contrast, a bi-exponential function with $T_{1}{ }^{\prime}$ of $0.848 \pm 0.007$ $\mathrm{s}$ and $T_{1}$ " of $0.003 \pm 0.009 \mathrm{~s}$ provides a superior fit for the relaxation behaviour of the second resonance centered at $12.6 \mathrm{ppm}$. The necessity of a bi-exponential fit reveals that the 12.6 ppm resonance actually represents two independent components from two different chemical environments in this powder sample. It is thus postulated that three separate $B$ environments exist in the $\mathrm{Pd}$-int $\mathrm{B} / \mathrm{C}$ system which contribute to the observed ${ }^{11} \mathrm{~B}$ MAS NMR spectrum. Two components have similar relaxation rates $\left(T_{1} \approx 1 \mathrm{~s}\right)$ whereas the third component relaxes much more rapidly $\left(T_{1}<0.01\right.$ s).

Furthermore, it is proposed that the fast-relaxing component of the $12.6 \mathrm{ppm}$ is the bulky interstitial B species (Table S5), as B positions inside the Pd metal lattice will be influenced by the conduction band electrons above the Fermi level which will subsequently induce very fast $T_{1}$ relaxation (see Figure 3c) ${ }^{43}$. This contrasts with the B species located on the $\mathrm{Pd} / \mathrm{C}$ surface (represented by the $0.7 \mathrm{ppm}$ resonance), where the loss of translational symmetry induces disruption to the density of states of the electronic conduction band, therefore reducing the efficiency of metallic relaxation. The slow relaxing component of the $12.6 \mathrm{ppm}$ resonance is attributed to sub-surface B species which experience a similar shift to the bulk B species, but the disruption in symmetry to the next-nearest-neighbor positions induces a similar perturbations to the density of states thereby resulting in less efficient metallic relaxation. Due to the electronic contact experienced by B in bulk and subsurface interstitial positions within the Pd metal structure, it is anticipated that the $12.6 \mathrm{ppm}$ shift is representative of a small ${ }^{11} \mathrm{~B}$ metallic Knight shift. Small ${ }^{11} \mathrm{~B}$ Knight shifts in various metallic environments have been previously reported with a measured shift of $12.6 \mathrm{ppm}$ from this system falling within the literature reported range ${ }^{44}$.

Previous solid-state NMR studies have demonstrated that a single environment which exhibits a strong quadrupole interaction can yield a bi-exponential relaxation profile 45 . To investigate the plausibility of this scenario, and to substantiate the assignments reported above, an ${ }^{11} \mathrm{~B}$ MAS NMR study of an acid-washed $\mathrm{Pd}$-intB/C system was undertaken. It is expected that dilute $\mathrm{HCl}$ wash should reduce the surface and sub-surface B components, leaving the bulk B interstitial component intact. However, the dilute acid washing has greatly reduced the intensity of all ${ }^{11} \mathrm{~B}$ MAS NMR resonances, therefore demonstrating an effect on the surface, sub-surface and the bulk interstitial B speciation. As observed in the Figure $3 \mathrm{~b}$, the overall intensity of the acid washed data has been greatly reduced so that $\sim 60$ times the number of transients is required to achieve a comparable $\mathrm{S} / \mathrm{N}$ ratio to that of the unwashed (original) $\mathrm{Pd}-\mathrm{int}^{\mathrm{B}} \mathrm{B} / \mathrm{C}$ system. At these reduced $\mathrm{S} / \mathrm{N}$ levels a full $T_{1}$ experimental analysis of the relaxation characteristics of the acid-washed Pdint $\mathrm{B} / \mathrm{C}$ system is not feasible; however, an abbreviated version allowing an estimation of the $T_{1}$ values can be achieved. As exhibited in Figure $3 \mathrm{~d}$, the relaxation rate can be projected by varying the recycle delay of a multi-transient single pulse experiment and observing $\mathrm{S} / \mathrm{N}$ (and relative speciation concentration) changes. If the recycle delay is less than five times the characteristic $T_{1}$ of the acid washed PdintB/C, then saturation effects will occur reducing the $S / N$ of the data (and possibly changing the relative intensities of the measured resonances). The results of Figure $3 \mathrm{~d}$ show that for the $12.6 \mathrm{ppm}$ resonance increasing the recycle delay from $0.1 \mathrm{~s}$ up to $5 \mathrm{~s}$ does not induce any change to the overall $\mathrm{S} / \mathrm{N}$ suggesting that the $T_{1}$ of this resonance must be $<0.02$ $\mathrm{s}$. This result confirms the existence of a fast-relaxing component within the $12.6 \mathrm{ppm}$ resonance of the unwashed $\mathrm{Pd}$ intB/C system, corroborating the necessity for a bi-exponential fit for the relaxation profile of this resonance. Furthermore, as the fast-relaxing component in this sample is not completely removed by the dilute $\mathrm{HCl}$ acid wash (conversely the slower sub-surface relaxing component is mostly removed), this fast-relaxing component is confidently attributed to the octahedral interstitial site within the bulk Pd metal framework.

Recent reports have shown that ${ }^{105} \mathrm{Pd}$ solid state NMR can be a sensitive probe of very small deviations from FCC structural symmetry in Pd metal systems ${ }^{18}$. Hence, the newly reported ${ }^{105} \mathrm{Pd}$ VOCS static NMR methodology was applied to the $\mathrm{Pd}$-int $\mathrm{B} / \mathrm{C}$ system and its undoped $\mathrm{Pd} / \mathrm{C}$ precursor, to investigate the local distortion of the Pd lattice induced by interstitial B. To aid in the acquisition of ${ }^{105} \mathrm{Pd} N M R, \mathrm{Pd}-$ int $\mathrm{B} / \mathrm{C}$ was synthesized from a Pd/C precursor with $45 \mathrm{wt} \%$ loading (see SI). These higher loaded samples exhibited particle sizes of $13 \pm 9 \mathrm{~nm}$, as determined by TEM. Figure 3e shows the ${ }^{105} \mathrm{Pd}$ static NMR data of the $\mathrm{Pd}$-int $\mathrm{B} / \mathrm{C}$ and $\mathrm{Pd} / \mathrm{C}$ systems directly compared to two previously established Pd metal systems (44-149 $\mu \mathrm{m}$ sized Pd metal particles and monodisperse $16 \pm 3 \mathrm{~nm}$ sized poly( $N$-vinyl-2-pyrrolidone) (PVP) stabilized Pd NPs) ${ }^{18}$. The micron sized Pd metal particles are characterised by a well-defined resonance at a Knight shift ( $\left.K_{\text {tot}}\right)$ of $-32,050 \pm 60 \mathrm{ppm}$; however, industrially relevant $\mathrm{Pd}$ NP systems such as PVP stabilized Pd and Pd/C of similar dimensions exhibit two markedly different characteristics $^{46}$. Firstly, the centre-of-gravity $K_{\text {tot }}$ shift of these resonances has shifted downfield to a 'less metallic'/reduced Knight shifted environment; and secondly, the resonance linewidth is $\sim 50$ times broader. The very large ${ }^{105} \mathrm{Pd}$ quadrupole moment $(Q)$ of the renders the quadrupole interaction at each Pd position to be a very sensitive probe of structural distortion and deviation from rigid FCC symmetry. 
There is an estimated $\sim 350,000-400,000$ atoms in cubooctahedral arrangements of $\mathrm{Pd}-{ }^{\text {int }} \mathrm{B} / \mathrm{C}$ and $\mathrm{Pd} / \mathrm{C}$ NPs of these reported dimensions, and it is evident that in both cases the whole nanoparticle is slightly perturbed from pure FCC symmetry due to the high surface-to-bulk atomic ratio. Although diffraction provides a longer range (periodic) average structure that suggests FCC symmetry is maintained, the short-range information from ${ }^{105} \mathrm{Pd}$ NMR suggests that particle size effects induce strain and disorder that introduces short range departure from this symmetry. In both systems there is no evidence of any strictly ordered FCC metal component represented by the narrow $K_{\text {tot }}-32,050$ \pm 60 ppm resonance. From Figure $3 \mathrm{~d}$ it can be observed that the incorporation of $\mathrm{B}$ into the $\mathrm{Pd} / \mathrm{C}$ system (yielding $\mathrm{Pd}$ intB) has a more marked effect on the ${ }^{105} \mathrm{Pd}$ static NMR spectrum. In addition to high surface area-to-bulk phenomenon for the particle size perturbing the Pd positions, the additional disorder generated by interstitial incorporation of the $\mathrm{B}$ increases the disorder broadening of the ${ }^{105} \mathrm{Pd}$ resonance to the extent where it cannot be observed (see the bottom of Figure $3 \mathrm{e}$ ). This inability to observe the ${ }^{105} \mathrm{Pd}$ signal upon B incorporation provides further confirmation of successful interstitial modification. If the $B$ remained only on the surface of the $\mathrm{Pd} / \mathrm{C}$ nanoparticles, then the NMR spectrum would resemble that of the $\mathrm{Pd} / \mathrm{C}$ precursor.

To this point, the combined suite of characteristic techniques has demonstrated that the proposed crystal structure for Pd-intB/C NPs exhibits overall FCC character, however the irregular B incorporation in the octahedral interstitial sites introduces short range disorder which is additional to that induced by particle size phenomena.

Local information analysis.

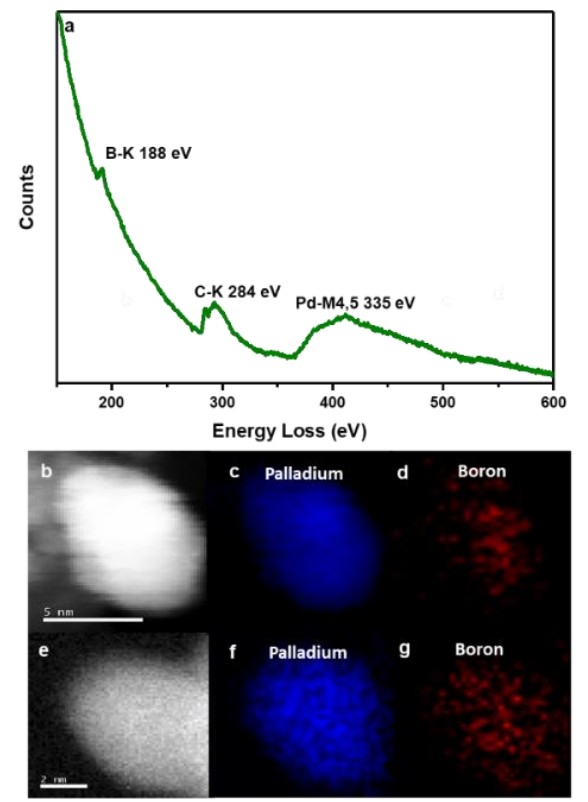

Figure 4. EELS analysis of a $\mathrm{Pd}-\mathrm{int} \mathrm{B} / \mathrm{C}$ sample. (a) A typical EELS spectrum image recorded from a Pd-intB NP. The three edges at $188 \mathrm{eV}, 284 \mathrm{eV}$ and $335 \mathrm{eV}$ correspond to the literature values of $\mathrm{B}-\mathrm{K}, \mathrm{C}-\mathrm{K}$ and $\mathrm{Pd}-\mathrm{M}_{4,5}$ edges respectively. (b) and (e) show STEM images of Pd-intB NPs. Elemental maps of the Pd-intB/C NPs are shown in (c), (d), (f) and (g), where the $\mathrm{Pd}-\mathrm{M}_{4,5}$ is labelled in blue and the B-K edge is labelled in red, respectively. The elemental distribution from the B- edge in (d) differs from that in (g), indicating that the doping source distributes unevenly for various NPs.

Despite the high resolution of ${ }^{11} \mathrm{~B}$ solid NMR, which confirmed the presence of interstitial $B$, it was also found that $\mathrm{Pd}$-intB/C NPs contain surface B species. In order to conduct a more local analysis of the elemental composition, STEM imaging was carried out in combination with EELS. In STEM-EELS, a high energy electron beam bombards with the specimen, and the energy lost by the inelastic scattered electrons, also called ionization edge, is used to characterize the elements present in the specimen. In particular, the fine structure of the ionization edge reveals chemical bonding and the oxidation state of the local environment.

A typical EELS spectrum and STEM image recorded while scanning the beam over one particle are shown in Figure 4a and Figure 4b. Further higher resolution STEM image and local elemental analysis of the particle are also shown in Figures 4c-g. The three main edges at $188 \mathrm{eV}, 284 \mathrm{eV}$ and $335 \mathrm{eV}$ coincide with literature values reported for the B-K edge $^{47}$, the $\mathrm{C}-\mathrm{K}$ edge ${ }^{48}$ and the $\mathrm{Pd}-\mathrm{M}_{4,5}$ edge $^{49}$ respectively. No evidence of oxygen was detected which would be manifested in an additional signal at $532 \mathrm{eV}^{50}$. Furthermore, peaks at $195 \mathrm{eV}$ and $202.5 \mathrm{eV}$, which correspond to features of $\mathrm{B}_{2} \mathrm{O}_{3}$ were not displayed. ${ }^{51}$ This demonstrates that locally NPs do not suffer from B surface contamination and that they are air stable at room temperature. The EELS elemental mapping from different NPs also showed that B doping does not distribute uniformly over the volume of the NPs, likely due to the low temperature deposition (Figure $4 \mathrm{c}, \mathrm{d}, \mathrm{f}, \mathrm{g}$ and supplementary information). In addition, STEM-EELS can provide local elemental analysis of individual NPs via spectra quantification. According to this analysis, as depicted in the Supplementary Information Table S7, the average $\mathrm{Pd}$ : $\mathrm{B}$ atomic composition ratio was approximately 1:0.2, similarly to that of the FCC $\mathrm{Pd}_{0.82} \mathrm{~B}_{0.18}$ phase ${ }^{52}$. The corresponding B site occupancy was therefore taken as 0.2 .

Ptychography insight into the nanostructure using the Wigner distribution deconvolution method. Being a phase imaging technique, ptychography is highly sensitive to light elements, such as B, and is therefore applicable to this system. This is clearly shown in supplementary information, Table S6 and Table S7 that the ptychographic phase was calculated for a range of typical NPs thicknesses and dopant occupancy. In practice, the highly defective nature of the nanoparticles may hinder contrast interpretation and the direct image of $B$ in the ptychographic phase. When crystal defects lie on planes that are parallel to the viewing direction, their presence is obvious. When inclined to the viewing direction the projected lattice will give rise to atomic-column peaks from atoms of the host Pd lattice between those from the FCC structure of a single crystal because the lattice is no longer view on a single projection vector. A series of atomic twins, extrinsic and intrinsic faults models along parallel $\mathrm{Pd}\{110\}$ planes that are inclined relative to the electron beam were constructed to identify the locations where addition peaks may rise. As shown in the Supplementary Information, from Figures S6 to S10, these atomic models did not show atomic occupancy at the observed octahedral site columns, but gave peaks closer to the tetrahedral interstitial sites. The presence of the crystallographic faults 
and the additional peaks arise at positions that would correspond to the tetrahedral interstitial sites.

Figure 5a, shows a typical STEM-ADF image obtained from a less defect NP. Because of the strong atomic number dependence of STEM-ADF $\left(Z_{P d}=46, Z_{C}=6\right.$ and $\left.Z_{B}=5\right)$, only $\mathrm{Pd}$ atoms are clearly seen as bright spots in the image, with several twinning planes visible (Figure $5 b$ ). To analyze the ADF contrast quantitatively with composition, a comparison with image calculations was conducted at the local concentration obtained by STEM-EELS. Simulated ADF images of $\mathrm{Pd}$ and $\mathrm{Pd}$-int $\mathrm{B} / \mathrm{C}$ are indistinguishable (Images are displayed in the Supplementary Information Figure S11 for a range of different crystal thicknesses).

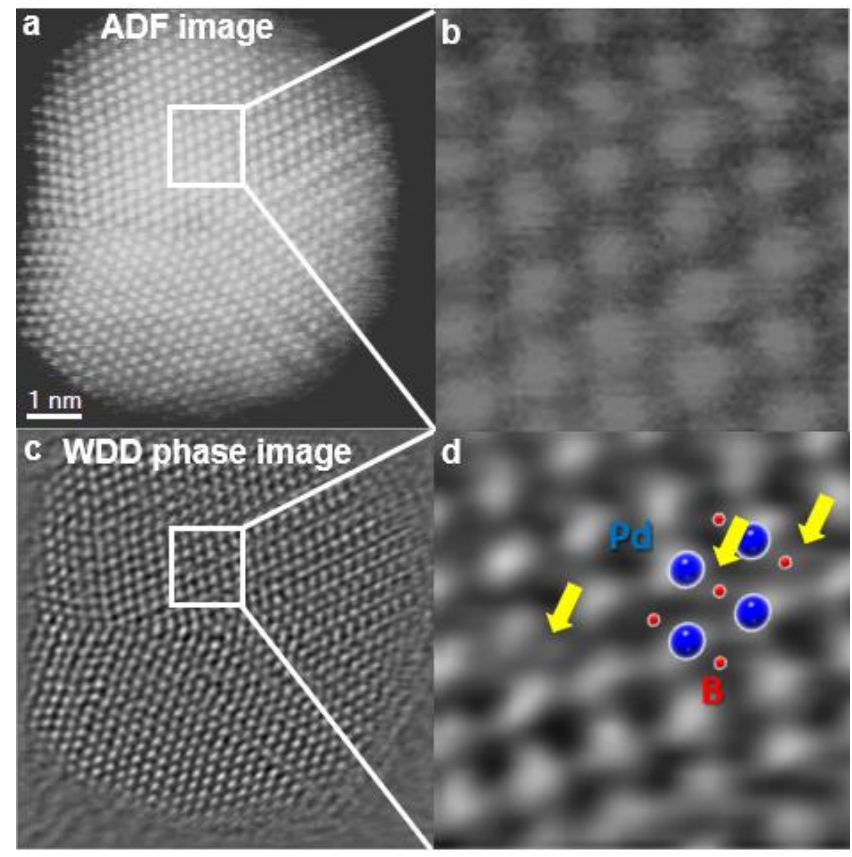

Figure 5. Simultaneously acquired Z-contrast STEM-ADF and ptychographic phase images of a Pd-intB/C NP oriented in the [110] zone axis. (a) Z-contrast STEM-ADF image of a Pd-intB/C NP. (b) inset from (a) shows an enlarged area of the NP. (c) Ptychographic phase image reconstructed using a Wigner Distribution Deconvolution (WDD) method after aberration correction ${ }^{53}$. (d) inset from (c) shows an enlarged area of the NP. The enlarged image shows some peaks visible between the Pd columns, which can be superimposed to the model predicted by XPDF.

At this concentration, the phase image should however provide a clear evidence for B contrast (see calculations in Figure S12). Restored ptychographic phase images are shown in Figure. 5c. Unlike the ADF Figure 5b, the ptychographic phase image after aberration correction (Figure 5c) clearly shows some peaks in interstitial octahedral sites between $\mathrm{Pd}$ atoms. If the projected $\mathrm{Pd}$-intB structure (see supplementary information Figure S5) is superimposed to enlarged image in Figures 5d, a good match is found at the octahedral sites. Combined with the data from the quantitative chemical techniques, the ptychography images can be explained by locations where a sufficiently high number of octahedral sites are filled by $B$ to give a peak in the phase image (Figure S13). Further evidence for B is shown in additional dataset in supplementary information Figure S14.

\section{CONCLUSION}

Supported metal particle of different sizes/shapes/defects modified by light interstitial atoms is commonly happened to industrial catalysts (small contaminants such as hydrogen, carbon or boron atoms can migrate into metal deliberately or non-deliberately to modify their catalytic properties at high calcination during synthesis. Such modification can affect the local structure at both short range and long range of the particles but unfortunately the interstitial effect is not evenly distributed throughout the metal particle. In summary, we have introduced a novel combination of high-resolution techniques beyond traditional characterization tools, which allow state-of-the-art assessment of macro and local structural changes when light elements as small as B atoms are unevenly doped in the sub-surface interstitial sites of supported noble metal NPs in industrial type powder catalysts. The multi-macroscopic analyses provide elemental distributions, crystal structures and establish general crystal models but local microanalyses can give local structural changes such as distorted sites, defective regions and local phase transformations, etc. Such broad range of analyses open a door for in-depth investigation of light element doped catalyst systems which give more relevant information to correlate their catalytic performance and guide further synthesis. Thus, a specific $\mathrm{Pd}$-intB/C NPs catalyst is hereby characterized from macro to microscale. Based on the characterization of multi-quantitative techniques, the atomic columns visualized by the fast pixelated ptychography technique could be ascribed to boron, which can create considerable local expansion to the metal lattice giving local lattice strain and phase transformation within a single Pd particle. Our future work will target to distinguish the atomic columns precisely within interior of metal framework, combined with atomic scale spectroscopic technique.

\section{ASSOCIATED CONTENT}

Supporting Information. This material is available free of charge via the Internet at http://pubs.acs.org." Experimental details, nanomaterials characterization spectra and additional analysis are all included.

\section{AUTHOR INFORMATION}

\author{
Corresponding Author \\ * edman.tsang@chem.ox.ac.uk (lead correspondence) \\ peter.nellist@material.ac.uk \\ J.V.Hanna@warwick.ac.uk
}

\section{ACKNOWLEDGMENT}

The financial support of EPSRC and Johnson Matthey (JM) are acknowledged. The authors also acknowledge dedicated EPSRC grant (EP/K040375/1) for South of England Analytical Electron Microscope [ATEM], EPSRC grant EP/M010708/1 and would also like to thank staff from Diamond Light Source, UK (I11 and I15) for access to beam-times. Tianyi Chen also wishes to thank Mr. James Sodo of Oxford Materials for his hyperspy coding for EELS and Dr. Aakash Varambhia for his kindly teaching and support. JVH thanks the University of Warwick and the Birmingham Science City Program for partial funding of the solid-state NMR infrastructure at Warwick. The latter program 
accessed the Birmingham Science City Advanced Materials Project 1: Creating and Characterising Next Generation Advanced Materials, which derived support from Advantage West Midlands (AWM) and the European Regional Development Fund (ERDF).

\section{REFERENCES}

(1) Duan, Y.; Song, T.; Dong, X.; Yang, Y. Enhanced Catalytic Performance of Cobalt Nanoparticles Coated with a N,P-Codoped Carbon Shell Derived from Biomass for Transfer Hydrogenation of Functionalized Nitroarenes. Green Chem. 2018, 20 (12), 2821-2828.

https://doi.org/10.1039/C8GC00619A.

(2) Tan, K. F.; Chang, J.; Borgna, A.; Saeys, M. Effect of Boron Promotion on the Stability of Cobalt Fischer-Tropsch Catalysts. J. Catal. 2011, 280 (1), 50-59.

https://doi.org/10.1016/j.jcat.2011.03.002.

(3) Han, M.-K.; Jin, Y.-S.; Yu, B. K.; Choi, W.; You, T.-S.; Kim, S.-J. Sulfur to Oxygen Substitution in BiOCuSe and Its Effect on the Thermoelectric Properties. J. Mater. Chem. A 2016, 4 (36), 13859-13865.

https://doi.org/10.1039/C6TA04310K.

(4) Teschner, D.; Borsodi, J.; Wootsch, A.; Revay, Z.; Havecker, M.; Knop-Gericke, A.; Jackson, S. D.; Schlogl, R. The Roles of Subsurface Carbon and Hydrogen in Palladium-Catalyzed Alkyne Hydrogenation. Science (80-. ). 2008, 320 (5872), 86-89.

https://doi.org/10.1126/science.1155200.

Okitsu, K.; Mizukoshi, Y.; Bandow, H.; Yamamoto, T. A.; Nagata, Y.; Maeda, Y. Synthesis of Palladium Nanoparticles with Interstitial Carbon by Sonochemical Reduction of Tetrachloropalladate(II) in Aqueous Solution. J. Phys. Chem. B 1997, 101 (28), 5470-5472. https://doi.org/10.1021/jp970415f.

(6) Li, Y.; Li, Z.; Ahsen, A.; Lammich, L.; Mannie, G. J. A.; Niemantsverdriet, J. W. H.; Lauritsen, J. V. Atomically Defined Iron Carbide Surface for Fischer-Tropsch Synthesis Catalysis. ACS Catal. 2019, 9 (2), 1264-1273.

https://doi.org/10.1021/acscatal.8b03684.

(7) Mavrikakis, M.; Hammer, B.; Nørskov, J. K. Effect of Strain on the Reactivity of Metal Surfaces. Phys. Rev. Lett. 1998, 81 (13), 2819-2822. https://doi.org/10.1103/PhysRevLett.81.2819.

(8) Wang, C.; Bartholomew, C. H. Preparation and Adsorption, Chemical and Catalytic Properties of Iron Boride Fischer-Tropsch Catalysts. Appl. Catal. 1990, 62 (1), 221-251. https://doi.org/10.1016/S01669834(00)82249-6.

(9) Zhou, Y.; Che, F.; Liu, M.; Zou, C.; Liang, Z.; De
Luna, P.; Yuan, H.; Li, J.; Wang, Z.; Xie, H.; Li, H.; Chen P.; Bladt, E.; Quintero-Bermudez, R.; Sham, T. K.; Bals, S.; Hofkens, J.; Sinton, D.; Chen, G.; Sargent, E. H. Dopant-Induced Electron Localization Drives CO2 Reduction to C2 Hydrocarbons. Nat. Chem. 2018, 10 (9), 974-980. https://doi.org/10.1038/s41557-018-0092-x.

(10) Trovarelli, A.; Llorca, J. Ceria Catalysts at Nanoscale: How Do Crystal Shapes Shape Catalysis? ACS Catal. 2017, 7 (7), 4716-4735. https://doi.org/10.1021/acscatal.7b01246.

Lynch, J.; Giannini, C.; Cooper, J. K.; Loiudice, A.; Sharp, I. D.; Buonsanti, R. Substitutional or Interstitial Site-Selective Nitrogen Doping in TiO 2 Nanostructures. J. Phys. Chem. C 2015, 119 (13), 7443-7452. https://doi.org/10.1021/jp512775s.

Su, D. S.; Zhang, B.; Schlögl, R. Electron Microscopy of Solid Catalysts-Transforming from a Challenge to a Toolbox. Chem. Rev. 2015, 115 (8), 2818-2882.

https://doi.org/10.1021/cr500084c.

Zhu, Y.; Ramasse, Q. M.; Brorson, M.; Moses, P. G.; Hansen, L. P.; Kisielowski, C. F.; Helveg, S. Visualizing the Stoichiometry of Industrial-Style Co-Mo-S Catalysts with Single-Atom Sensitivity. Angew. Chemie Int. Ed. 2014, 53 (40), 1072310727.

https://doi.org/10.1002/anie.201405690.

(14) Yang, B.; Burch, R.; Hardacre, C.; Hu, P.; Hughes, P. Selective Hydrogenation of Acetylene over PdBoron Catalysts: A Density Functional Theory Study. J. Phys. Chem. C 2014, 118 (7), 3664-3671. https://doi.org/10.1021/jp412255a.

(15) Hoffmann, K.; Hooper, T. J. N.; Murshed, M. M.; Dolotko, O.; Révay, Z.; Senyshyn, A.; Schneider, H.; Hanna, J. V.; Gesing, T. M.; Fischer, R. X. Formation, Stability and Crystal Structure of Mullite-Type Al 6-x B x 0 9. J. Solid State Chem. 2016, 243, 124-135.

https://doi.org/10.1016/j.jssc.2016.08.018.

(16) Hoffmann, K.; Hooper, T. J. N.; Zhao, H.; Kolb, U.; Murshed, M. M.; Fischer, M.; Lührs, H.; Nénert, G.; Kudějová, P.; Senyshyn, A.; Schneider, H.; Hanna, J. V.; Gesing, Th. M.; Fischer, R. X. Crystal Chemical Characterization of Mullite-Type Aluminum Borate Compounds. J. Solid State Chem. 2017, 247, 173-187.

https://doi.org/10.1016/j.jssc.2016.12.027.

(17) Park, D.-S.; Rees, G. J.; Wang, H.; Rata, D.; Morris, A. J.; Maznichenko, I. V.; Ostanin, S.; Bhatnagar, A.; Choi, C.-J.; Jónsson, R. D. B.;Kaufmann, K.; Kashtiban, R.; Walker, M.; Chiang, C.; Thorsteinsson, E. B.; Luo, Z.; Park, I.; Hanna, J. V.; Mertig, I.; Dörr, K.; Gíslason H. P.; McConville, C. F. Electromagnetic Functionalization of Wide- 
Bandgap Dielectric Oxides by Boron Interstitial Doping. Adv. Mater. 2018, 30 (39), 1802025. https://doi.org/10.1002/adma.201802025.

(18) Hooper, T. J. N.; Partridge, T. A.; Rees, G. J.; Keeble, D. S.; Powell, N. A.; Smith, M. E.; Mikheenko, I. P.; Macaskie, L. E.; Bishop, P. T.; Hanna, J. V. Direct Solid State NMR Observation of the $105 \mathrm{Pd}$ Nucleus in Inorganic Compounds and Palladium Metal Systems. Phys. Chem. Chem. Phys. 2018, 20 (41), 26734-26743.

https://doi.org/10.1039/C8CP02594K.

(19) Lin, F.; Liu, Y.; Yu, X.; Cheng, L.; Singer, A.; Shpyrko, O. G.; Xin, H. L.; Tamura, N.; Tian, C.; Weng, T.-C.; Yang, X.; Meng, Y. S.; Nordlund, D.; Yang, W.; Doeff, M. M.. Synchrotron X-Ray Analytical Techniques for Studying Materials Electrochemistry in Rechargeable Batteries. Chem. Rev. 2017, 117 (21), 13123-13186. https://doi.org/10.1021/acs.chemrev.7b00007.

(20) Pennycook, S. J.; Chisholm, M. F.; Lupini, A. R.; Varela, M.; Borisevich, A. Y.; Oxley, M. P.; Luo, W. D.; van Benthem, K.; Oh, S.-H.; Sales, D. L.;Molina, S. I.; García-Barriocanal, J.; Leon, C.; Santamaría, J.; Rashkeev, S. N.; Pantelides, S. T. AberrationCorrected Scanning Transmission Electron Microscopy: From Atomic Imaging and Analysis to Solving Energy Problems. Philos. Trans. R. Soc. A Math. Phys. Eng. Sci. 2009, 367 (1903), 37093733. https://doi.org/10.1098/rsta.2009.0112.

(21) Hiley, C. I.; Playford, H. Y.; Fisher, J. M.; Felix, N. C.; Thompsett, D.; Kashtiban, R. J.; Walton, R. I. Pair Distribution Function Analysis of Structural Disorder by $\mathrm{Nb} 5+$ Inclusion in Ceria: Evidence for Enhanced Oxygen Storage Capacity from Under-Coordinated Oxide. J. Am. Chem. Soc. 2018, 140 (5), 1588-1591.

https://doi.org/10.1021/jacs.7b12421.

(22) Egerton, R. F. Electron Energy-Loss Spectroscopy in the Electron Microscope, 3rd ed.; Springer: New York, 2011. https://doi.org/10.1007/978-14419-9583-4.

(23) Yang, H.; Rutte, R. N.; Jones, L.; Simson, M.; Sagawa, R.; Ryll, H.; Huth, M.; Pennycook, T. J.; Green, M. L. H.; Soltau, H.; Kondo, Y.; Davis, B. G.; Nellist, P. D. Simultaneous Atomic-Resolution Electron Ptychography and Z-Contrast Imaging of Light and Heavy Elements in Complex Nanostructures. Nat. Commun. 2016, 7 (1), 12532. https://doi.org/10.1038/ncomms12532.

(24) Jiang, K.; Xu, K.; Zou, S.; Cai, W. B - Doped Pd Catalyst: Boosting Room-Temperature Hydrogen Production from Formic Acid - Formate Solutions. J. Am. Chem. Soc. 2014, 136, 4861-4864. https://doi.org/dx.doi.org/10.1021/ja5008917.

(25) Ellis, I. T.; Wolf, E. H.; Jones, G.; Lo, B.; Meng-Jung
Li, M.; York, A. P. E.; Tsang, S. C. E. Lithium and Boron as Interstitial Palladium Dopants for Catalytic Partial Hydrogenation of Acetylene. Chem. Commun. 2017, 53 (3), 601-604. https://doi.org/10.1039/C6CC08404D.

Chan, C. W. A.; Mahadi, A. H.; Li, M. M.-J.; Corbos, E. C.; Tang, C.; Jones, G.; Kuo, W. C. H.; Cookson, J.; Brown, C. M.; Bishop, P. T.; Tsang, S. C. E. Interstitial Modification of Palladium Nanoparticles with Boron Atoms as a Green Catalyst for Selective Hydrogenation. Nat. Commun. 2014, 5, 5787.

https://doi.org/10.1038/ncomms6787.

(27) Batchellor, A. S.; Kwon, G.; Laskowski, F. A. L.; Tiede, D. M.; Boettcher, S. W. Domain Structures of $\mathrm{Ni}$ and NiFe (Oxy)Hydroxide Oxygen-Evolution Catalysts from X-Ray Pair Distribution Function Analysis. J. Phys. Chem. C 2017, 121 (45), 2542125429.

https://doi.org/10.1021/acs.jpcc.7b10306.

(28) Petkov, V.; Parvanov, V.; Trikalitis, P.; Malliakas, C.; Vogt, T.; Kanatzidis, M. G. Three-Dimensional Structure of Nanocomposites from Atomic Pair Distribution Function Analysis: Study of Polyaniline and (Polyaniline) $0.5 \mathrm{~V} 205 \cdot 1.0 \mathrm{H} 2$ O. J. Am. Chem. Soc. 2005, 127 (24), 8805-8812. https://doi.org/10.1021/ja051315n.

(29) Harrington, R.; Hausner, D. B.; Bhandari, N.; Strongin, D. R.; Chapman, K. W.; Chupas, P. J.; Middlemiss, D. S.; Grey, C. P.; Parise, J. B. Investigation of Surface Structures by Powder Diffraction: A Differential Pair Distribution Function Study on Arsenate Sorption on Ferrihydrite. Inorg. Chem. 2010, 49 (1), 325-330. https://doi.org/10.1021/ic9022695.

Reeder, R. J.; Tang, Y.; Schmidt, M. P.; Kubista, L. M.; Cowan, D. F.; Phillips, B. L. Characterization of Structure in Biogenic Amorphous Calcium Carbonate: Pair Distribution Function and Nuclear Magnetic Resonance Studies of Lobster Gastrolith. Cryst. Growth Des. 2013, 13 (5), 19051914. https://doi.org/10.1021/cg301653s.

(31) Jones, L.; Yang, H.; Pennycook, T. J.; Marshall, M. S. J.; Van Aert, S.; Browning, N. D.; Castell, M. R.; Nellist, P. D. Smart Align - a New Tool for Robust Non-Rigid Registration of Scanning Microscope Data. Adv. Struct. Chem. Imaging 2015, 1 (1), 8. https://doi.org/10.1186/s40679-015-0008-4.

(32) Pennycook, T. J.; Lupini, A. R.; Yang, H.; Murfitt, M. F.; Jones, L.; Nellist, P. D. Efficient Phase Contrast Imaging in STEM Using a Pixelated Detector. Part 1: Experimental Demonstration at Atomic Resolution. Ultramicroscopy 2015, 151, 160-167. https://doi.org/10.1016/j.ultramic.2014.09.013
Lozano, J. G.; Martinez, G. T.; Jin, L.; Nellist, P. D.; Bruce, P. G. Low-Dose Aberration-Free Imaging 
of Li-Rich Cathode Materials at Various States of Charge Using Electron Ptychography. Nano Lett. 2018, 18 (11), 6850-6855.

https://doi.org/10.1021/acs.nanolett.8b02718.

(34) Raine, E.; Clark, A. H.; Smales, G.; Smith, A.; Gianolio, D.; Li, T.; Zheng, J.; Griffith, B. E.; Hyde, T. I.; Feaviour, M.; Collier, P.; Hanna, J. V.; Sankar, G.; Tsang, S. C. E. Synthesis and Characterization of Platinum Nanoparticle Catalysts Capped with Isolated Zinc Species in SBA-15 CChannels: The Wall Effect. ACS Appl. Nano Mater. 2018, 1 (12), 6603-6612.

https://doi.org/10.1021/acsanm.8b01357.

(35) Navaladian, S.; Viswanathan, B.; Varadarajan, T. K.; Viswanath, R. P. A Rapid Synthesis of Oriented Palladium Nanoparticles by UV Irradiation. Nanoscale Res. Lett. 2009, 4 (2), 181-186. https://doi.org/10.1007/s11671-008-9223-4.

(36) Chan, C. W. A.; Xie, Y.; Cailuo, N.; Yu, K. M. K.; Cookson, J.; Bishop, P.; Tsang, S. C. New Environmentally Friendly Catalysts Containing Pd-Interstitial Carbon Made from Pd-Glucose Precursors for Ultraselective Hydrogenations in the Liquid Phase. Chem. Commun. 2011, 47 (28), 7971-7973.

https://doi.org/10.1039/c1cc12681d.

(37) Pennycook, T. J.; Jones, L.; Pettersson, H.; Coelho, J.; Canavan, M.; Mendoza-Sanchez, B.; Nicolosi, V.; Nellist, P. D. Atomic Scale Dynamics of a Solid State Chemical Reaction Directly Determined by Annular Dark-Field Electron Microscopy. Sci. Rep. 2015, 4 (1), 7555. https://doi.org/10.1038/srep07555.

(38) Kobayashi, K.; Kobayashi, H.; Maesato, M.; Hayashi, M.; Yamamoto, T.; Yoshioka, S.; Matsumura, S.; Sugiyama, T.; Kawaguchi, S.; Kubota, Y.; Nakanishi, H.; Kitagawa, H. Discovery of Hexagonal Structured Pd-B Nanocrystals. Angew. Chemie - Int. Ed. 2017, 56 (23), 65786582. https://doi.org/10.1002/anie.201703209.

(39) Coelho, A. A. TOPAS and TOPAS-Academic: An Optimization Program Integrating Computer Algebra and Crystallographic Objects Written in C++. J. Appl. Crystallogr. 2018, 51 (1), 210-218. https://doi.org/10.1107/S1600576718000183.

(40) Dutta, B. N.; Dayal, B. Lattice Constants and Thermal Expansion of Palladium and Tungsten up to $878^{\circ} \mathrm{C}$ by X-Ray Method. Phys. status solidi 1964, 5 (1), 73-75.

https://doi.org/10.1002/pssb.19640050108.

(41) Bakhmutov, V. I. Practical NMR Relaxation for Chemists; John Wiley \& Sons, Ltd: Chichester, UK, 2004. https://doi.org/10.1002/0470094486.

(42) Narayanan, A.; Hartman, J. S.; Bain, A. D. Characterizing Nonexponential Spin-Lattice Relaxation in Solid-State NMR by Fitting to the
Stretched Exponential. J. Magn. Reson. Ser. A 1995, 112 (1), 58-65.

https://doi.org/10.1006/jmra.1995.1009.

van der Klink, J. J.; Brom, H. B. NMR in Metals, Metal Particles and Metal Cluster Compounds. Prog. Nucl. Magn. Reson. Spectrosc. 2000, 36 (2), 89-201.https://doi.org/10.1016/S00796565(99)00020-5.

(44) Pavarini, E.; Baek, S. H.; Suh, B. J.; Borsa, F.; Bud ko, S. L.; Canfield, P. C. NMR Relaxation Rates and Knight Shifts in MgB 2 and AlB 2 : Theory versus Experiments. Supercond. Sci. Technol. 2003, 16 (2), 147-151. https://doi.org/10.1088/09532048/16/2/303.

(45) Yesinowski, J. P. Finding the True Spin-Lattice Relaxation Time for Half-Integral Nuclei with Non-Zero Quadrupole Couplings. J. Magn. Reson. 2015, 252, 135-144.

https://doi.org/10.1016/j.jmr.2014.12.012.

(46) Cookson, J. The Preparation of Palladium Nanoparticles. Platin. Met. Rev. 2012, 56 (2), 8398.

https://doi.org/10.1595/147106712X632415.

(47) Sato, K.; Fukata, N.; Hirakuri, K. Doping and Characterization of Boron Atoms in Nanocrystalline Silicon Particles. Appl. Phys. Lett. 2009, 94 (16), 161902.

https://doi.org/10.1063/1.3120768.

(48) Lieser, G.; Mittler-Neher, S.; Spinke, J.; Knoll, W. Electron Microscopic Investigations on FreeStanding Mixed Lipid Langmuir-Blodgett-Kuhn Monolayers: Phase Separation and Aging Process. Biochim. Biophys. Acta - Biomembr. 1994, $1192 \quad$ (1), 14-20. https://doi.org/10.1016/0005-2736(94)901376.

(49) Longo, P.; Twesten, R. D.; Kothleitner, G. Fast STEM EELS Spectrum Imaging Analysis of Pd-Au Based Catalysts. Microsc. Microanal. 2013, 19 (S2), 1612-1613.

https://doi.org/10.1017/S1431927613010052.

(50) C. C. Ans and O.L.Krivanek. EELS Atlas, New York, 1982.

(51) Nicholls, R. J.; Aslam, Z.; Sarahan, M. C.; Koo, A.; Yates, J. R.; Nellist, P. D.; Grobert, N. BoronMediated Nanotube. ACS Nano 2012, 6 (9), 78007805.

https://doi.org/doi.org/10.1021/nn301770b.

Beck, M.; Ellner, M.; Mittemeijer, E. J. Unit Cell Parameters and Densities of the Solid Solution Pd(B). Mater. Sci. Forum 2000, 321-324, 604609.

https://doi.org/10.4028/www.scientific.net/MS F.321-324.604.

(53) Yang, H.; MacLaren, I.; Jones, L.; Martinez, G. T.; 
Simson, M.; Huth, M.; Ryll, H.; Soltau, H.; Sagawa, R.; Kondo, Y.; Ophus, C.; Ercius, P.; Jin, L.; Kovács, A.;Nellist P. D.. Electron Ptychographic Phase Imaging of Light Elements in Crystalline Materials Using Wigner Distribution Deconvolution. Ultramicroscopy 2017, 180, 173-
179.

https://doi.org/10.1016/j.ultramic.2017.02.006 
Insert Table of Contents artwork here

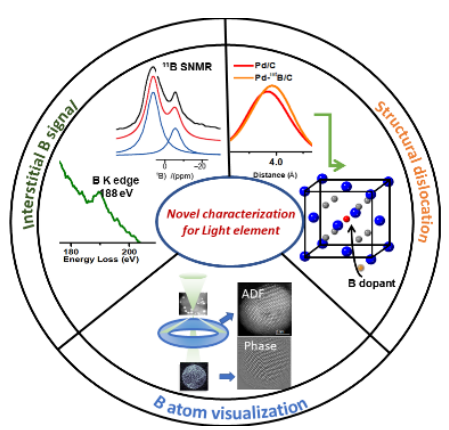

